The Astrophysical Journal, 558:L109-L112, 2001 September 10

(C) 2001. The American Astronomical Society. All rights reserved. Printed in U.S.A.

\title{
AFTERGLOW EMISSION FROM HIGHLY COLLIMATED JETS WITH FLAT ELECTRON SPECTRA: APPLICATION TO THE GRB 010222 CASE?
}

\author{
Z. G. DAI ${ }^{1}$ AND K. S. CHENG ${ }^{2}$ \\ Received 2001 May 3; accepted 2001 July 30; published 2001 August 10
}

\begin{abstract}
We derive light curves of the afterglow emission from highly collimated jets if the power-law index $(p)$ of the electron energy distribution is above 1 but below 2. We find the following: (1) Below the characteristic synchrotron frequency, the light-curve index depends generally on $p$. (2) As long as the jet expansion is spherical, the light-curve index above the characteristic frequency increases slowly as the spectral index of the emission increases. (3) Once the jet enters the spreading phase, the high-frequency emission flux decays as proportional to $t^{-(p+6) / 4}$ rather than proportional to $t^{-p}$. All these results differ from those in the case of $p>2$. We compare our analytical results with the observations on the GRB 010222 afterglow and conclude that the jet model may be unable to explain the observed data. Thus, a more promising explanation for this afterglow seems to be the expansion of a relativistic fireball or a mildly collimated jet in a dense medium.
\end{abstract}

Subject headings: gamma rays: bursts - relativity — shock waves

\section{INTRODUCTION}

Gamma-ray burst (GRB) afterglows are believed to be emitted from a relativistic shock wave expanding in its surrounding medium via synchrotron radiation or inverse Compton scattering (ICS) of accelerated electrons in the shocked matter (Piran 1999; van Paradijs, Kouveliotou, \& Wijers 2000; Cheng \& Lu 2001). To interpret the abundant data of afterglows, the effects of environments such as preburst stellar winds (Dai \& Lu 1998; Mészáros, Rees, \& Wijers 1998; Chevalier \& Li 1999, 2000) and dense media (Dai \& Lu 1999, 2000; Wang, Dai, \& $\mathrm{Lu} 2000$ ) have been discussed. On the other hand, jets are of particular interest because they have important implications on almost all aspects of the GRB phenomenon, e.g., the total energy that is released in an explosion, the event rate, the physical ejection mechanism, and the afterglow decay rate. The most exciting implication is that the transition of a relativistic jet to the spreading phase can result in steepening of the afterglow light curve to the flux proportional to $t^{-p}$, as analyzed by Rhoads (1999) and Sari, Piran, \& Halpern (1999). Following the analytical work, many numerical calculations have been performed, and they are essentially consistent with the analytical results (e.g., Panaitescu \& Mészáros 1999; Moderski, Sikora, \& Bulik 2000; Kumar \& Panaitescu 2000; Panaitescu \& Kumar 2001; Huang, Dai, \& Lu 2000a, 2000b; Huang et al. 2000c; Wei \& Lu 2000). The jet model seems to account well for a few well-observed afterglows with light-curve breaks, e.g., GRB 990123 (Kulkarni et al. 1999; Castro-Tirado et al. 1999; Fruchter et al. 1999), GRB 990510 (Harrison et al. 1999; Stanek et al. 1999), GRB 991216 (Halpern et al. 2000), GRB 000301C (Rhoads \& Fruchter 2001; Masetti et al. 2000; Jensen et al. 2001; Berger et al. 2000; Sagar et al. 2000), GRB 000418 (Berger et al. 2001), and GRB 000926 (Price et al. 2001; Harrison et al. 2001; Sagar et al. 2001a; Piro et al. 2001).

GRB 010222 is the latest well-observed burst, whose optical afterglow light curve has an earliest sharp break (Masetti et al. 2001; Stanek et al. 2001; Sagar et al. 2001b; Cowsik et al.

\footnotetext{
${ }^{1}$ Department of Astronomy, Nanjing University, 22 Hankou Lu, Nanjing, Jiangsu 210093, China; daizigao@public1.ptt.js.cn.

${ }^{2}$ Department of Physics, University of Hong Kong, Hong Kong, China; hrspksc@hkucc.hku.hk.
}

2001). A popular explanation is that this afterglow might have come from a highly collimated jet with a flat-spectrum electron distribution $(1<p<2)$. In this Letter, we derive light curves of the emission when such jets expand in an interstellar medium (ISM) or in a stellar wind and find that the jet model may be inconsistent with the afterglow data of GRB 010222.

\section{LIGHT CURVES}

Let us assume an adiabatic relativistic jet with an initial half-opening angle of $\theta_{0}$, a laterally spreading velocity of $c_{s}$, and a bulk Lorentz factor of $\gamma$. This assumption is valid if the energy density of the electrons accelerated by a shock, produced by the interaction of the jet with its surrounding medium, is a small fraction $\epsilon_{e}$ of the total energy density of the shocked medium or if most of the electrons are adiabatic, i.e., their radiative cooling timescale is larger than that of the jet expansion (Sari, Piran, \& Narayan 1998). The energy density carried by magnetic fields is assumed to be another fraction $\epsilon_{B}$ of the total energy density of the shocked medium, and thus the magnetic strength $B=\left[32 \pi \epsilon_{B} \gamma^{2} n(r) m_{p} c^{2}\right]^{1 / 2}$, where $m_{p}$ is the proton mass and $n(r)$ is the proton number density of the surrounding medium at shock radius $r$. We adopt a power-law density profile, $n(r)=A r^{-s}$, where $A=n_{*} \times 1 \mathrm{~cm}^{-3}$ for the ISM ( $s=0)$ and $A=3 \times 10^{35} A_{*} \mathrm{~cm}^{-1}$ for the wind ( $s=2$; Chevalier \& Li 1999).

We consider synchrotron radiation of the electrons accelerated by the shock. To calculate the spectrum and light curve, one needs to determine three break frequencies: the selfabsorption frequency $\left(\nu_{a}\right)$, the characteristic frequency $\left(\nu_{m}\right)$, and the cooling frequency $\left(\nu_{c}\right)$. The latter two frequencies can be directly derived from the minimum Lorentz factor $\gamma_{m}$ and the cooling Lorentz factor $\gamma_{c}$, which appear in the energy distribution of cooled electrons. As usual, we adopt a power-law injection of electrons with the energy distribution (just behind the shock front) given by $d n_{e} / d \gamma_{e} \propto \gamma_{e}^{-p}$ for $\gamma_{m} \leq \gamma_{e} \leq \gamma_{M}$, where $\gamma_{M}=\left[3 e /\left(\xi \sigma_{\mathrm{T}} B\right)\right]^{1 / 2}$ is the maximum electron Lorentz factor, which is calculated by assuming that the acceleration time equals the synchrotron cooling time. Here $\xi \sim 1$ is the ratio of the acceleration time to the gyration time, $e$ is the electron charge, and $\sigma_{\mathrm{T}}$ is the Thomson cross section. According to this electron energy distribution and the jump conditions for a relativistic shock, the electron number density and en- 
ergy density of the shocked medium can be written as two integrals: $\int_{\gamma_{m}}^{\gamma_{M}}\left(d n_{e} / d \gamma_{e}\right) d \gamma_{e}=4 \gamma n$ and $\int_{\gamma_{m}}^{\gamma_{M}}\left(\gamma_{e} m_{e} c^{2}\right)\left(d n_{e} / d \gamma_{e}\right) d \gamma_{e}=$ $4 \gamma^{2} n m_{p} c^{2} \epsilon_{e}$, where $m_{e}$ is the electron mass. Such integrals combined with the assumption of a flat electron spectrum $(1<$ $p<2$ ) lead to

$$
\gamma_{m}=\left(\frac{2-p}{p-1} \frac{m_{p}}{m_{e}} \epsilon_{e} \gamma \gamma_{M}^{p-2}\right)^{1 /(p-1)}
$$

Equation (1) is different from the frequently used minimum Lorentz factor $\gamma_{m}=[(p-2) /(p-1)]\left(m_{p} / m_{e}\right) \epsilon_{e} \gamma$, which is also derived from these integrals for $p>2$. Consequently, we will obtain expressions for afterglow light curves that differ from those derived by Sari et al. (1998, 1999). In addition, the age of the jet could also provide a limit on $\gamma_{m}$ through constraining $\gamma_{M}$ in equation (1). However, we have found that the value of $\gamma_{M}$ inferred from the age limit is usually much larger than the one from the limit that the acceleration time equals the synchrotron cooling time, and thus the age limit on $\gamma_{m}$ can be ignored. The value of $\gamma_{c}$, the Lorentz factor of electrons that cool on the expansion time, is given by $\gamma_{c}=$ $6 \pi m_{e} c /\left(\sigma_{\mathrm{T}} \gamma B^{2} t\right)$, where $t$ is the observer's time (neglecting the redshift correction; Sari et al. 1998). After having $\gamma_{m}$ and $\gamma_{c}$, we can easily obtain the evolution of $\nu_{m}$ and $\nu_{c}$ with time based on $\nu_{m} \propto \gamma \gamma_{m}^{2} B$ and $\nu_{c} \propto \gamma \gamma_{c}^{2} B$ (see below). The remaining break frequency is the self-absorption one, which is given by $\nu_{a}=$ $\left\{5 \text { enr } /\left[(3-s) B \gamma_{m}^{5}\right]\right\}^{3 / 5} \nu_{m}$ for $\nu_{a} \ll \nu_{m}<\nu_{c}$ (expected at late times of the afterglow; cf. Panaitescu \& Kumar 2000).

The next crucial question is how the Lorentz factor $\gamma$ decays with the observer's time because the break frequencies and the peak flux, which are needed in calculating the observed flux, are functions of $\gamma$ and of the shock radius and medium density. Even if the shock is beamed, as long as $\gamma>\theta_{0}^{-1}\left(c_{s} / c\right)$, the jet evolution is a spherelike expansion based on the Blandford \& McKee (1976) self-similar solution, and thus the Lorentz factor decreases as $\gamma=8.2\left(E_{53} / n_{*}\right)^{1 / 8} t^{-3 / 8}$ for $s=0$, or $\gamma=8.8\left(E_{53} / A_{*}\right)^{1 / 4} t^{-1 / 4}$ for $s=2$, where $E_{53}$ is the isotropicequivalent energy of the jet in units of $10^{53} \mathrm{ergs}$ and $t$ is in units of 1 day. However, the transition of the jet evolution takes place at $\gamma \sim \theta_{0}^{-1}\left(c_{s} / c\right)$, which, in fact, defines the break time $t_{b}$. After this time, the jet will enter the spreading phase with $c_{s}=c / \sqrt{3}$ in Rhoads (1999) or with $c_{s}=c$ in Sari et al. (1999). As a result, the Lorentz factor decays as $\gamma \propto t^{-1 / 2}$.

After knowing the evolution of $\gamma$, we can find scaling relations of the break frequencies with time. First, we derive the characteristic frequency

$$
\nu_{m} \propto \begin{cases}t^{-[3(p+2)] /[8(p-1)]}, & \text { spherical in ISM, } \\ t^{-(p+4) /[4(p-1)]}, & \text { spherical in wind, } \\ t^{-(p+2) /[2(p-1)]}, & \text { jet. }\end{cases}
$$

Second, the self-absorption frequency is found to evolve as

$$
\nu_{a} \propto \begin{cases}t^{[9(2-p)] /[16(p-1)]}, & \text { spherical in ISM, } \\ t^{(74-49 p) /[40(p-1)]}, & \text { spherical in wind, } \\ t^{(34-19 p) /[20(p-1)]}, & \text { jet. }\end{cases}
$$

Finally, the cooling frequency evolves as $\nu_{c} \propto t^{-1 / 2}$ for a spherical shock in the ISM, $\nu_{c} \propto t^{1 / 2}$ for a spherical shock in the wind, and $\nu_{c} \propto t^{0}$ for a jet.

In addition, the observed peak flux, $F_{\nu_{m}}$, has been derived by many authors (e.g., Waxman 1997; Dai \& Lu 1998; Wijers \& Galama 1999; Rhoads 1999; Sari et al. 1998, 1999; Chevalier \& Li 2000). In this Letter, we neglect the effect of dust ex- tinction on the peak flux because this effect has been discussed to be significant only for a highly collimated jet expanding in a dense circumstellar cloud by Dai, Huang, \& Lu (2001).

Therefore, we can calculate the light curves for four frequency ranges. The flux at frequencies lower than $\nu_{a}$, $F_{\nu<\nu_{a}}=F_{\nu_{m}}\left(\nu_{a} / \nu_{m}\right)^{1 / 3}\left(\nu / \nu_{a}\right)^{2}$ and thus evolves as

$$
F_{\nu<v_{a}} \propto \begin{cases}t^{(17 p-26) /[16(p-1)]}, & \text { spherical in ISM, } \\ t^{(13 p-18) /[8(p-1)]}, & \text { spherical in wind, } \\ t^{[3(p-2)] /[4(p-1)]}, & \text { jet. }\end{cases}
$$

The flux above the self-absorption frequency but below the characteristic frequency is given by $F_{\nu_{a}<\nu<\nu_{m}}=F_{\nu_{m}}\left(\nu / \nu_{m}\right)^{1 / 3}$, which evolves as

$$
F_{\nu_{a}<\nu<\nu_{m}} \propto \begin{cases}t^{(p+2) /[8(p-1)]}, & \text { spherical in ISM, } \\ t^{[5(2-p)] /[12(p-1)]}, & \text { spherical in wind, } \\ t^{(8-5 p) /[6(p-1)]}, & \text { jet. }\end{cases}
$$

It is seen from equations (4) and (5) that, below $\nu_{m}$, the lightcurve index is still determined by $p$. As a comparison, the index is independent of $p$ in the case of $p>2$ (Sari et al. 1999). For $p=1.5$ (similar to the index obtained by Malkov 1999 for Fermi acceleration in the limit when particles acquire a significant fraction of the shock energy), the flux at $\nu<\nu_{a}$ is approximately constant, and the flux at $\nu_{a}<\nu<\nu_{m}$ increases as proportional to $t^{7 / 8}$ for the ISM case and proportional to $t^{5 / 12}$ for the wind case, respectively, as long as the expansion is spherical. Then, once the jet enters the spreading phase, the flux below the self-absorption frequency begins to decline as proportional to $t^{-0.75}$, and the flux at higher frequency begins to increase slowly as proportional to $t^{1 / 6}$.

If the observed high-frequency emission comes from the radiating electrons that are slow cooling, we have its flux $F_{\nu_{m}<\nu<\nu_{c}}=F_{\nu_{m}}\left(\nu / \nu_{m}\right)^{-(p-1) / 2}$, which decays as

$$
F_{\nu_{m}<\nu<\nu_{c}} \propto \begin{cases}t^{-3(p+2) / 16}, & \text { spherical in ISM, } \\ t^{-(p+8) / 8}, & \text { spherical in wind, } \\ t^{-(p+6) / 4}, & \text { jet. }\end{cases}
$$

Above the cooling frequency, we obtain $F_{\nu>\nu_{c}}=F_{\nu_{m}} \times$ $\left(\nu_{c} / \nu_{m}\right)^{-(p-1) / 2}\left(\nu / \nu_{c}\right)^{-p / 2}$, which declines as

$$
F_{\nu>\nu_{c}} \propto \begin{cases}t^{-(3 p+10) / 16}, & \text { spherical in ISM, } \\ t^{-(p+6) / 8}, & \text { spherical in wind, } \\ t^{-(p+6 / 4}, & \text { jet. }\end{cases}
$$

Bhattacharya (2001) derived light curves of the emission from a jet expanding in the ISM by assuming a general case of $\gamma_{M} \propto \gamma^{q}$ in equation (1). Our light curves in equations (6) and (7) are consistent with his $q=-\frac{1}{2}$ result. We define the lightcurve index $\alpha$ and the spectral index $\beta$ through $F_{\nu}(t) \propto$ $t^{-\alpha} \nu^{-\beta}$. Table 1 summarizes the relations between $\alpha$ and $\beta$ above $\nu_{m}$ for different cases. Figures 1 and 2 further present the $\alpha-\beta$ relations for $1<p<2$ as well as those for $p>2$, in the ISM and wind cases, respectively. We see that, for each line in these figures, the $p<2$ segment is not an extrapolation of the $p>$ 2 segment.

\section{COMPARISON WITH THE AFTERGLOW OF GRB 010222}

The UBVRI light curve of the GRB 010222 afterglow has been fitted by one broken power law: $F_{\nu} \propto t^{-\alpha_{1}}$ before the break time $t_{b}$ and $F_{\nu} \propto t^{-\alpha_{2}}$ after $t_{b}$. Here we summarize the light- 
TABLE 1

Spectral Index $\beta$ and the Light-Curve Index $\alpha$ as Function of $p$ IN THE CASE OF $1<p<2$

\begin{tabular}{lllll}
\hline \hline & \multirow{2}{*}{ Spectral INDEX $\beta$} & \multicolumn{3}{c}{ Light-Curve Index $\alpha\left(F_{\nu} \propto t^{-\alpha}\right)$} \\
\cline { 3 - 5 } FrequenCy & \multicolumn{1}{c}{$\left(F_{\nu} \propto \nu^{-\beta}\right)$} & \multicolumn{1}{c}{ Sphere in ISM } & Sphere in Wind & Jet \\
\hline$\nu<\nu_{c} \ldots \ldots$ & $\beta=(p-1) / 2$ & $\alpha=3(p+2) / 16$ & $\alpha=(p+8) / 8$ & $(p+6) / 4$ \\
& $\beta=(p-1) / 2$ & $\alpha=3(2 \beta+3) / 16$ & $\alpha=(2 \beta+9) / 8$ & $(2 \beta+7) / 4$ \\
$\nu>\nu_{c} \ldots \ldots$ & $\beta=p / 2$ & $\alpha=(3 p+10) / 16$ & $\alpha=(p+6) / 8$ & $(p+6) / 4$ \\
& $\beta=p / 2$ & $\alpha=(3 \beta+5) / 8$ & $\alpha=(\beta+3) / 4$ & $(\beta+3) / 2$ \\
\hline
\end{tabular}

NoTE. - The parameter-free relation between $\alpha$ and $\beta$ is given for each case by eliminating $p$.

curve indices, the break time, and the spectral index given in the literature: $\left(\alpha_{1}, \alpha_{2}, t_{b}, \beta\right)$ are $(0.60 \pm 0.03,1.31 \pm 0.03$, $0.48 \pm 0.02$ days, $1.1 \pm 0.1$; Masetti et al. 2001), (0.80 \pm $0.05,1.30 \pm 0.05,0.72 \pm 0.10$ days, $0.88 \pm 0.10$; Stanek et al. 2001), and $(0.74 \pm 0.05,1.35 \pm 0.04,0.7 \pm 0.07$ days, $0.75 \pm 0.02$; Sagar et al. 2001b). In addition, the X-ray decay index after the break measured by BeppoSAX is $\alpha_{2}=$ $1.33 \pm 0.04$, and the spectral index $\beta=0.97 \pm 0.05$ (in 't Zand et al. 2001). A common result of the optical and X-ray observations is that the light curve indeed began to steepen proportional to $t^{-1.3}$ about 0.5 days after the GRB. This is the earliest observed break of all the studied afterglows.

The temporal property of the afterglow from GRB 010222 is naturally reminiscent of the jet model. Indeed, some authors (e.g., Stanek et al. 2001; Sagar et al. 2001b; Cowsik et al. 2001) attributed this afterglow to a highly collimated jet. Stanek et al. gave a spectral fit of their BVRI data and obtained an index of $\beta=0.88 \pm 0.10$, in excellent agreement with the $g^{\prime} r^{\prime} i^{\prime} z^{\prime}$ fit of Lee et al. (2001), $\beta=0.90 \pm 0.03$, and with the spectral index given by Jha et al. (2001), $\beta=0.89 \pm 0.03$. This implies a spectral index of the electron distribution of $p=2.8$ in the slow-cooling electron regime or $p=1.8$ in the fast-cooling electron regime. The former value of $p$ leads to $F_{\nu} \propto t^{-2.8}$ at late times (Rhoads 1999; Sari et al. 1999), while the latter value gives $F_{v} \propto t^{-1.95}$ (see $\S 2$ ). These results are inconsistent with the observed late-time light curve $\left(\propto t^{-1.3}\right)$. Stanek et al. have noted this inconsistency. To save the jet model, they suggested that the spectral index could be intrinsically in the range of $0.5<\beta<0.7$ due to the SMC-like ex-

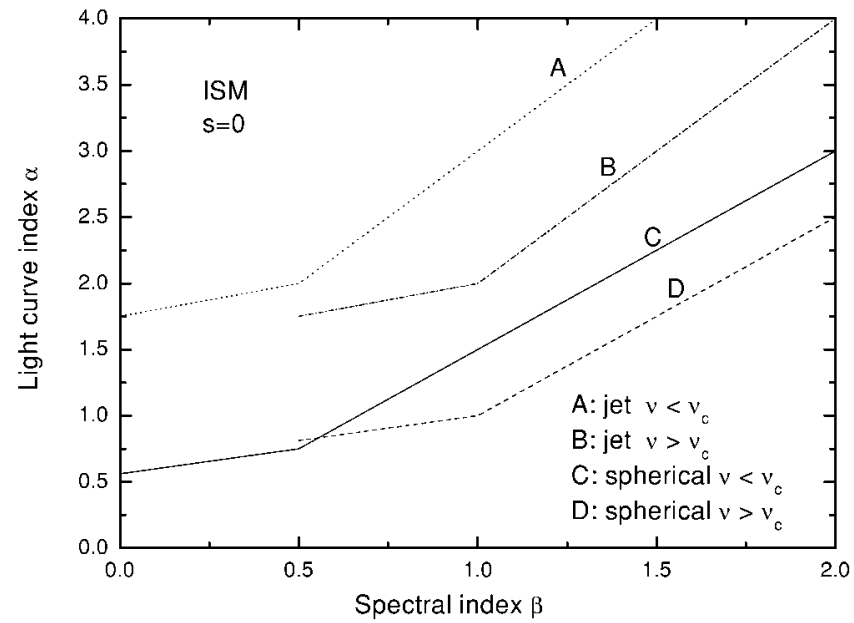

FIG. 1.-Plot of the light-curve index $(\alpha)$ vs. the spectral index $(\beta)$ in the ISM case. Lines A and B correspond to a highly collimated but spreading jet whose (observed) high-frequency emission comes from the radiating electrons that are slow cooling $\left(\nu<\nu_{c}\right)$ and fast cooling $\left(\nu>\nu_{c}\right)$, respectively, and lines $\mathrm{C}$ and $\mathrm{D}$ correspond to a spherical shock. tinction. Such a range of the spectral index requires $2<p<$ 2.4 (slow cooling) or $1<p<1.4$ (fast cooling). Even if the value of $p$ becomes smaller for the theoretical spectral index to be compatible with the observed extinction-corrected spectral index, according to Rhoads (1999), Sari et al. (1999), and our analysis in $\S 2$, we still conclude that the spreading jet model cannot provide an explanation for the late-time lightcurve index. We note that Sagar et al. (2001b) suggested the afterglow of GRB 010222 as evidence for a highly collimated jet with a fast-cooling, flat-spectrum electron distribution. Their argument is that the emission flux from a spreading jet decays as proportional to $t^{-p}$ for $1<p<2$, which means $F_{\nu} \propto t^{-1.3}$ when $p=1.3$, inferred by their fitting spectrum. However, from our analysis in $\S 2$, we see that their argument is incorrect.

An alternative explanation for the afterglow of GRB 010222 is the expansion of a relativistic fireball or a mildly collimated jet in a medium with density of $10^{5}-10^{6} \mathrm{~cm}^{-3}$ (Masetti et al. 2001; in 't Zand et al. 2001). In such a dense medium, the fireball decelerates to the nonrelativistic regime within a few days after the burst, resulting in a steepening of the light curve (Dai \& Lu 1999, 2000). In 't Zand et al. argued that the nonrelativistic interpretation with a universal $p \approx 2.2$ value is consistent with the observations. They also noted that the dense-medium assumption is compatible with the observed redshift-corrected column density of $\sim 2.5 \times 10^{22} \mathrm{~cm}^{-2}$.

\section{DISCUSSION AND CONCLUSION}

We have derived light curves of the emission when a highly collimated jet with a flat-spectrum electron distribution $(1<$ $p<2$ ) expands in the ISM or in the preburst wind. The most important finding of ours is that once the jet begins to spread, the light-curve index becomes $(p+6) / 4$ rather than $p$. There-

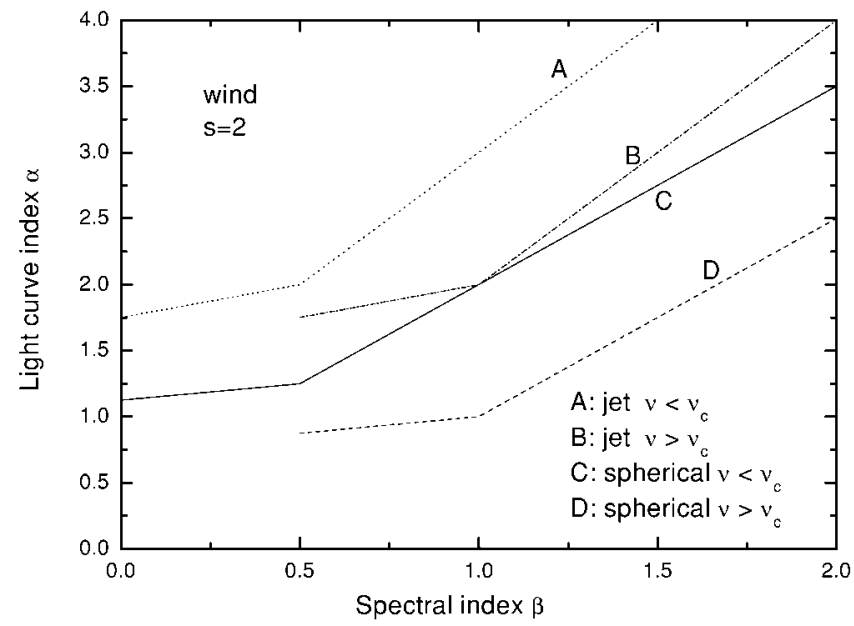

FIG. 2.- Same as Fig. 1, but in the wind case 
fore, the jet model appears to be inconsistent with the afterglow data of GRB 010222. ICS in the shocked medium does not influence the light curves derived in $\S 2$. This is because for $p<2$ most of the electron energy behind the shock front should be radiated away via both synchrotron radiation and ICS and thus the Compton parameter $Y \approx\left[-1+\left(1+4 \epsilon_{e} / \epsilon_{B}\right)^{1 / 2}\right] / 2$ is approximately constant (Panaitescu \& Kumar 2000; Sari \& Esin 2001).

Two important quantities that future observations led by HETE-2 and Swift will provide are the light-curve index and the spectral index, which, once known, will show a point in Figures 1 and 2. According to the position of this point in these figures, one will be able to not only obtain information on the dynamical evolution of a postburst shock wave and the radiation regime of the accelerated electrons (slow cooling or fast cooling) but also infer the value of $p$.

It should be emphasized that our derivations in $\S 2$ are based on the assumption that the electron energy density behind a shock is a constant fraction $\left(\epsilon_{e}\right)$ of the total energy density of the shocked medium, as used in the standard afterglow shock model. If this assumption is invalid, the minimum Lorentz factor of the electrons, without any acceleration, could become $\gamma$ instead of equation (1). In such a case, the previous jet model could explain the afterglow of GRB 010222 if the electron energy distribution is required to be a power law with $p<2$. However, it is unclear whether this requirement is satisfied in the absence of any acceleration.

We are very grateful to the referee and D. M. Wei for valuable comments that significantly improved the manuscript and to $\mathrm{N}$. Masetti and B. Zhang for discussions. This work was supported by an RGC grant of the Hong Kong government, the National Natural Science Foundation of China (grant 19825109), and the National 973 Project (NKBRSF G19990754).

\section{REFERENCES}

Berger, E., et al. 2000, ApJ, 545, 56 2001, ApJ, in press (astro-ph/0102278)

Bhattacharya, D. 2001, Bull. Astron. Soc. India, 29, 107

Blandford, R. D., \& McKee, C. F. 1976, Phys. Fluids, 19, 1130

Castro-Tirado, A. J., et al. 1999, Science, 283, 2069

Cheng, K. S., \& Lu, T. 2001, Chinese Astron. Astrophys., 1, 1

Chevalier, R. A., \& Li, Z. Y. 1999, ApJ, 520, L29 2000, ApJ, 536, 195

Cowsik, R., et al. 2001, Bull. Astron. Soc. India, in press (astro-ph/0104363)

Dai, Z. G., Huang, Y. F., \& Lu, T. 2001, MNRAS, 324, L11

Dai, Z. G., \& Lu, T. 1998, MNRAS, 298, 87 . 1999, ApJ, 519, L155 2000, ApJ, 537, 803

Fruchter, A. S., et al. 1999, ApJ, 519, L13

Halpern, J. P., et al. 2000, ApJ, 543, 697

Harrison, F. A., et al. 1999, ApJ, 523, L121 2001, ApJ, in press (astro-ph/0103377)

Huang, Y. F., Dai, Z. G., \& Lu, T. 2000a, MNRAS, 316, 943 . 2000b, A\&A, 355, L43

Huang, Y. F., Gou, L. J., Dai, Z. G., \& Lu, T. 2000c, ApJ, 543, 90

in 't Zand, J. J. M., et al. 2001, ApJ, in press (astro-ph/0104362)

Jensen, B. L., et al. 2001, A\&A, 370, 909

Jha, S., et al. 2001, ApJ, 554, L155

Kulkarni, S. R., et al. 1999, Nature, 398, 389

Kumar, P., \& Panaitescu, A. 2000, ApJ, 541, L9

Lee, B. C., et al. 2001, ApJ, in press (astro-ph/0104201)

Malkov, M. 1999, ApJ, 511, L53
Masetti, N., et al. 2000, A\&A, 359, L23 2001, A\&A, 374, 382

Mészáros, P., Rees, M. J., \& Wijers, R. A. M. J. 1998, ApJ, 499, 301

Moderski, R., Sikora, M., \& Bulik, T. 2000, ApJ, 529, 151

Panaitescu, A., \& Kumar, P. 2000, ApJ, 543, 66 2001, ApJ, 554, 667

Panaitescu, A., \& Mészáros, P. 1999, ApJ, 526, 707

Piran, T. 1999, Phys. Rep., 314, 575

Piro, L., et al. 2001, ApJ, in press (astro-ph/0103306)

Price, P. A., et al. 2001, ApJ, 549, L7

Rhoads, J. 1999, ApJ, 525, 737

Rhoads, J., \& Fruchter, A. S. 2001, ApJ, 546, 117

Sagar, R., et al. 2000, Bull. Astron. Soc. India, 28, 499 2001a, Bull. Astron. Soc. India, 29, 1 2001b, Bull. Astron. Soc. India, 29, 91

Sari, R., \& Esin, A. A. 2001, ApJ, 548, 787

Sari, R., Piran, T., \& Halpern, J. P. 1999, ApJ, 519, L17

Sari, R., Piran, T., \& Narayan, R. 1998, ApJ, 497, L17

Stanek, K. Z., et al. 1999, ApJ, 522, L39 . 2001, ApJL, submitted (astro-ph/0104329)

van Paradijs, J., Kouveliotou, C., \& Wijers, R. A. M. J. 2000, ARA\&A, 38, 379

Wang, X. Y., Dai, Z. G., \& Lu, T. 2000, MNRAS, 317, 170

Waxman, E. 1997, ApJ, 485, L5

Wei, D. M., \& Lu, T. 2000, ApJ, 541, 203

Wijers, R. A. M. J., \& Galama, T. J. 1999, ApJ, 523, 177 